\title{
Présentation des appareils de mesure des débits et volumes d'eau
}

\author{
A. Costes \\ SAGEP (Eaux de Paris)
}

\section{Le cas de la Ville de Paris}

A deux reprises, pour améliorer la gestion des réseaux, le service des eaux de la Ville de Paris a eu recours aux dispositifs de comptage.

Après 1880 , la généralisation des compteurs d'abonnés a permis non seulement de connaître et facturer les volumes consommés mais, également, de limiter le gaspillage.

A partir de 1985, la privatisation du service a nécessité d'installer des débitmètres aux points de fourniture de l'eau en gros. Ces dispositifs ont conduit à déterminer avec précision les rendements des réseaux et abouti à leur amélioration. La figure I précise quelques chiffres.

Cette expérience parisienne nous donne l'occasion de dégager les tendances actuelles en matière de débitmétrie.

\section{Le choix entre compteurs et débitmètres}

\subsection{La difficulté du choix}

Si l'utilité du comptage ne se discute plus, le choix des appareils n'est pas toujours aisé.

Dans la grande majorité des cas on fait appel aux compteurs d'eau mécaniques car ils sont économiques à l'achat, de précision garantie par directive CEE et de fonctionnement éprouvé. Ils sont donc utilisés pour la vente de l'eau aux abonnés sous le contrôle du Service National de Métrologie Légale.

En revanche, diverses conditions particulières obligent à recourir aux débitmètres ; on peut citer notamment :

- le comptage en grands diamètres $(>500 \mathrm{~mm})$;

- les équipements temporaires lors de campagnes de mesure ;

- les débits très élevés, très faibles, réversibles ;

- la qualité de l'eau lorsqu'elle ne convient pas aux systèmes mécaniques.

Il est donc de tradition d'opposer les compteurs aux débitmètres. Si les premiers sont réglementés, ce qui a pour conséquence d'entraver leur progrès technique, les seconds, normalisés pour une partie d'entre eux seulement, requièrent de la part de l'utilisateur de la vigilance au moment du choix.

Ce choix est d'autant plus vaste que, sur l'eau, tous les débitmètres usuels fonctionnent et peuvent notamment être étalonnés sur ce liquide. Dernière difficulté pratique, aucun constructeur ne propose objectivement la totalité des systèmes. Par ailleurs, certains utilisateurs, déçus autrefois par les performances insuffisantes de matériels électroniques proposés prématurément, restent prudents.

\subsection{Les critères de choix}

Choisir un matériel destiné au comptage de l'eau nécessite d'examiner les critères suivants :

- les coûts d'achat, d'installation, d'exploitation et de maintenance,

- la qualité, fiabilité et garantie de la mesure,

- l'environnement hydraulique (longueurs droites requises, qualité de l'eau, perte de charge).

Auparavant, la connaissance des divers systèmes disponibles pour l'eau (leur nombre dépasse la vingtaine) conduit à opérer une distinction fondamentale entre compteurs, débitmètres et méthodes (fig. 2). Le but de cette classification, détaillée en figure 2 bis, est d'amener l'utilisateur à observer deux points essentiels.

1 - Une chaîne de mesure doit être identifiée dans sa totalité, du tronçon de conduite à l'affichage de la grandeur mesurée.

2 - Le procédé de mesure doit être connu. Il faut observer en priorité si la mesure porte sur l'ensemble de la section (relevé de vitesse moyenne: cas des électromagnétiques ou turbines), sur une partie (ultrasons, sondes électromagnétiques) ou sur une vitesse ponctuelle.

$\mathrm{Si}$ les traitements actuels des signaux numériques n'amènent pratiquement aucune erreur sur la conversion du signal en volume ou débit, que ce signal soit une différence de temps, une tension électrique, une pression différentielle, une vibration, etc... (on pourra rencontrer des débitmètres dont la mesure est gérée par un système informa-

\section{Introduction to the measurement of water flows and volumes}

The author presents the different kinds of flowmeters and water volume measurement devices with their advantages and drawbacks and their typical applications. 


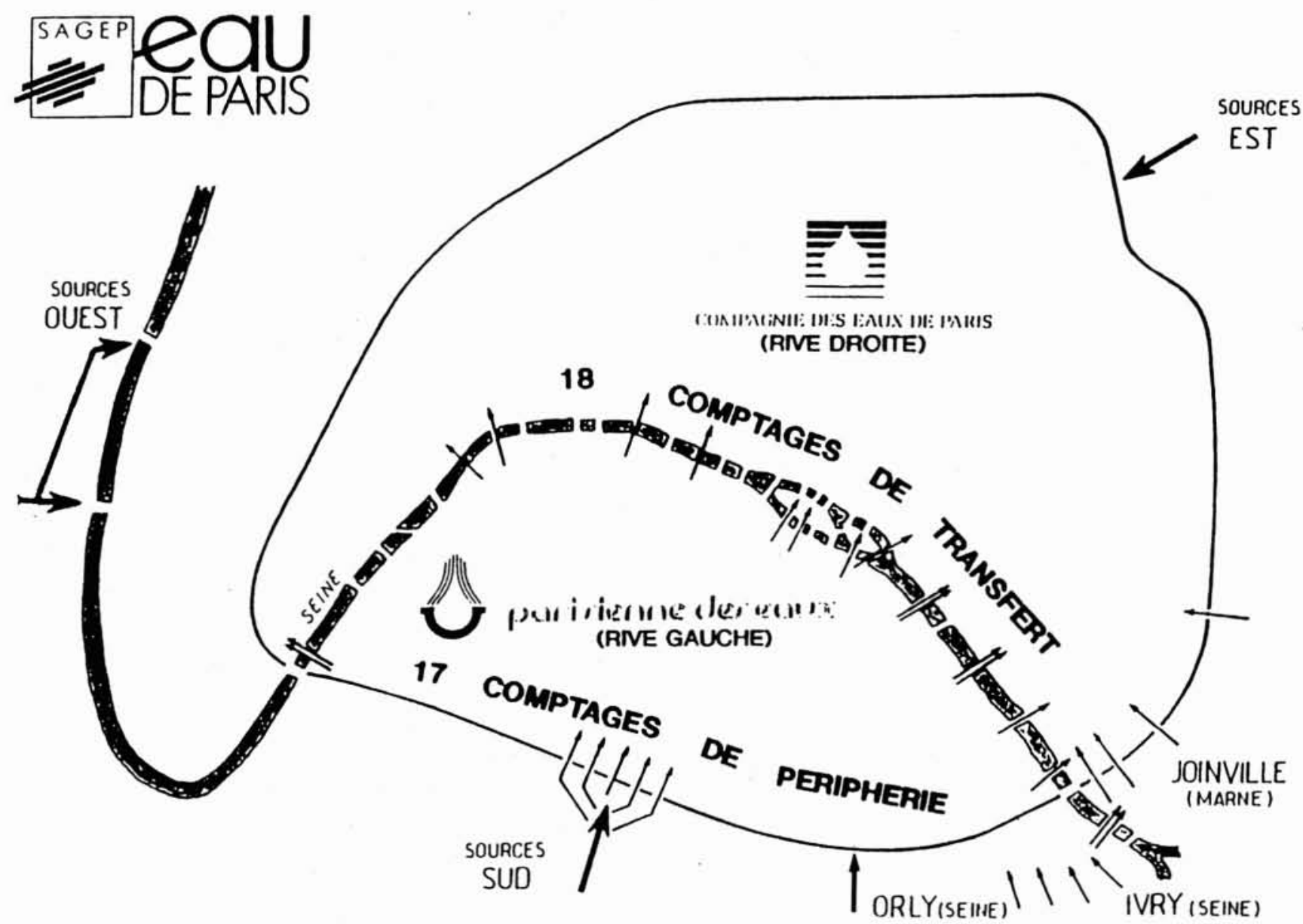

1. Les comptages parisiens.

Production SAGEP 1993: $262 \mathrm{Mm}^{3}$.

Consommations abonnés : $237 \mathrm{Mm}^{3}$.

Rendement : 0,90.

Prix de l'eau à Paris au 1.01.1994:

Vente en gros (SAGEP) : 2,346 F HT.

Distribution (CEP-SPE) : 2,126 F HT.

Vente au détail : 4,472 F HT.

Total Abonné TTC: 11,111 F.

tisé), il ne faudra jamais oublier que cette aide ne dispense pas de connaître la forme de l'écoulement et la géométrie de la section de mesure.

\subsection{A propos de précision et installation des appareils}

Il est d'usage d'inclure dans une classification un tableau résumant les précisions et conditions d'installations des appareils. Des éléments chiffrés sur ces caractéristiques étant souvent discutés et parfois discutables, il est préférable de donner des conseils pouvant aider à leur détermination.

\subsubsection{La précision}

L'erreur de mesure d'un compteur ou débitmètre doit être définie sans ambiguïté : sur quelle grandeur s'applique-telle ?, par rapport à quoi est-elle exprimée (pourcentage de la valeur mesurée ou du fond d'échelle?). Les valeurs maximales tolérées sur cette erreur doivent être fixées sur une dynamique de mesure mentionnée.

Que le matériel soit neuf ou en service, il doit toujours être possible d'en connaître la précision.

\subsubsection{Les conditions d'installations}

Dans les appareils qui déterminent une vitesse d'eau dans une section de conduite, les écoulements doivent être normalisés (axisymétriques); des longueurs droites amont et aval de tuyauteries sont nécessaires.

Dans les conditions de référence, les bancs d'étalonnage ne doivent introduire aucune influence. En service, les parties droites peuvent être raccourcies car on accepte une légère dérive de l'erreur de mesure.

Sont sensibles aux profils de vitesse les appareils qui mesurent sur une partie d'écoulement (plan diamétral, mesure ponctuelle...) surtout en présence de perturbations sévères (coudes non coplanaires, pompes, vannes partiellement fermées...). 

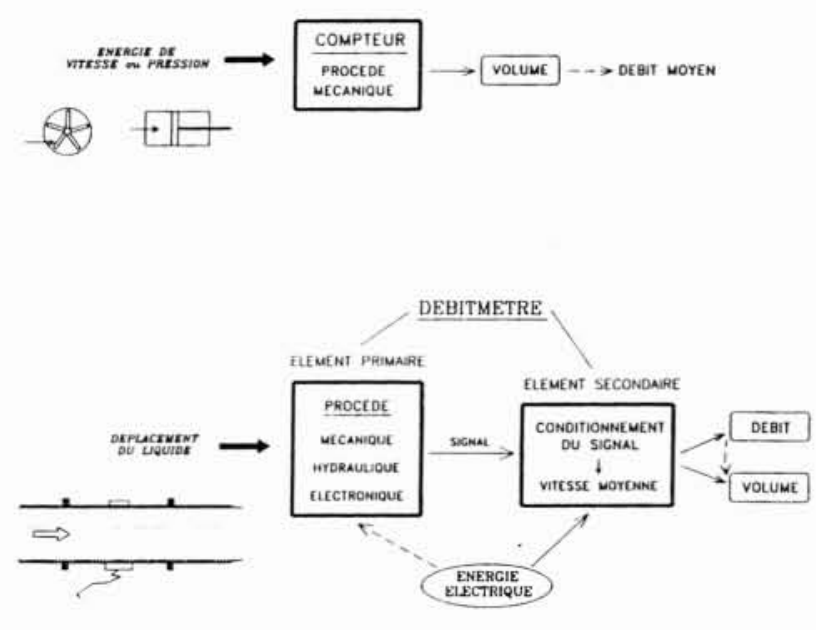

METHODES

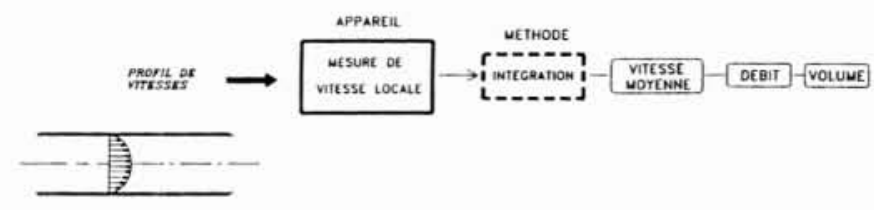

2. Types de mesure des débits ou volumes d'eau.
Enfin, les dispositifs tranquiliseurs corrigent généralement la précision des appareils et non la qualité des écoulements.

\section{Principaux dispositifs de comptage}

\subsection{Compteur volumétrique}

Métrologiquement le meilleur, car ses chambres mesureuses déterminent en continu des volumes cycliques. Difficile à construire en gros diamètre (maximum actuel $65 \mathrm{~mm}$ ), il s'installe en toute position mais n'aime ni les eaux chargées ni les débits élevés permanents.

\subsection{Compteurs à turbine}

Apparemment simple mécaniquement. Sa précision s'est améliorée sous l'effet des réglementations CEE et approche celle des compteurs volumétriques si les conditions d'installations sont respectées (longueurs droites amont aval; cadran du compteur horizontal). Préférer le jet unique au compteur à jets multiples dont les trous d'injection et de réglage peuvent se colmater si l'eau est déposante.

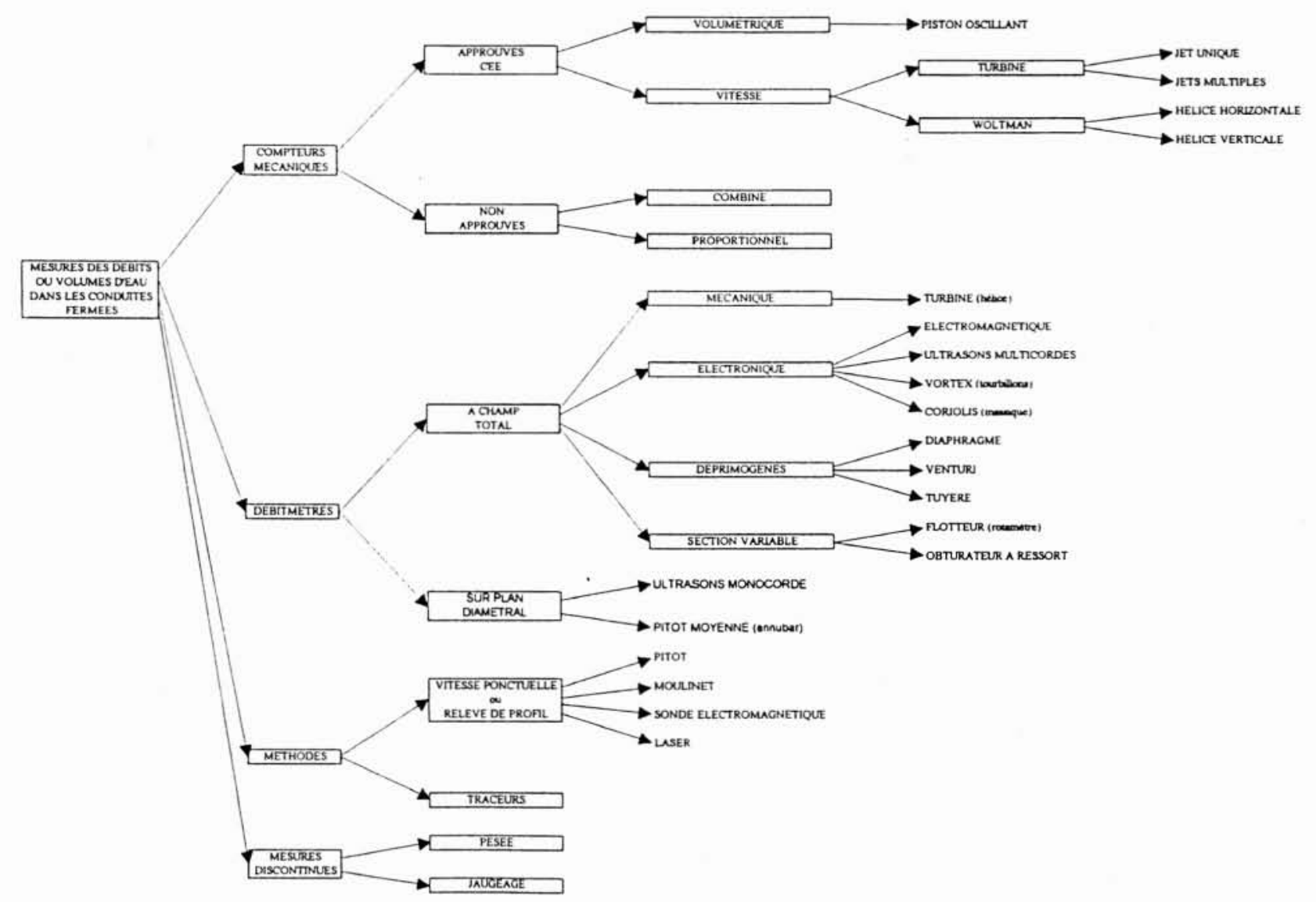

2 bis. Classification des appareils de mesure. 
VOLUMETRIQUE A PISTON ROTATIF.
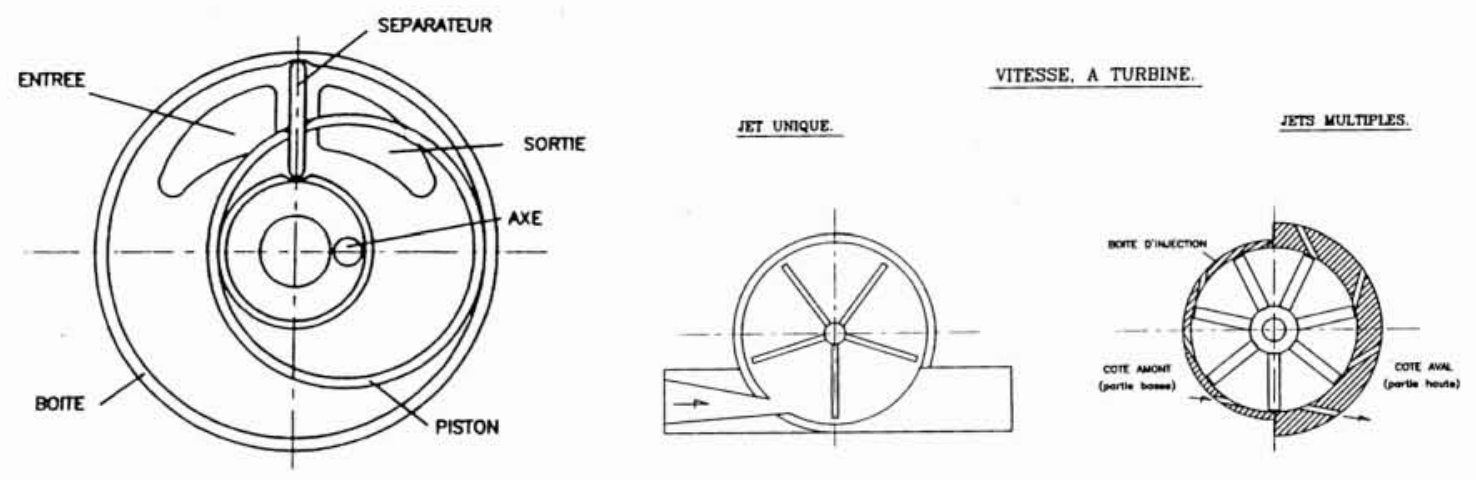

3. Compteurs mécaniques, petits diamètres.

\section{WOLTMAN}

AXIAL, amovible

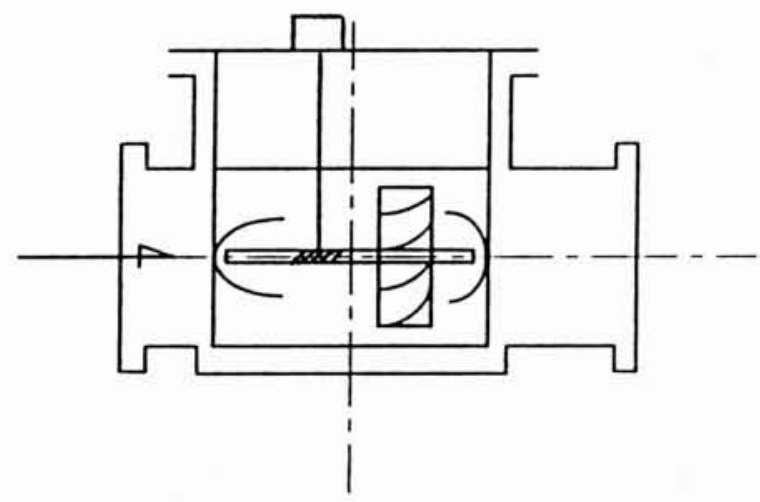

COMPTEUR PROPORTIONNEL

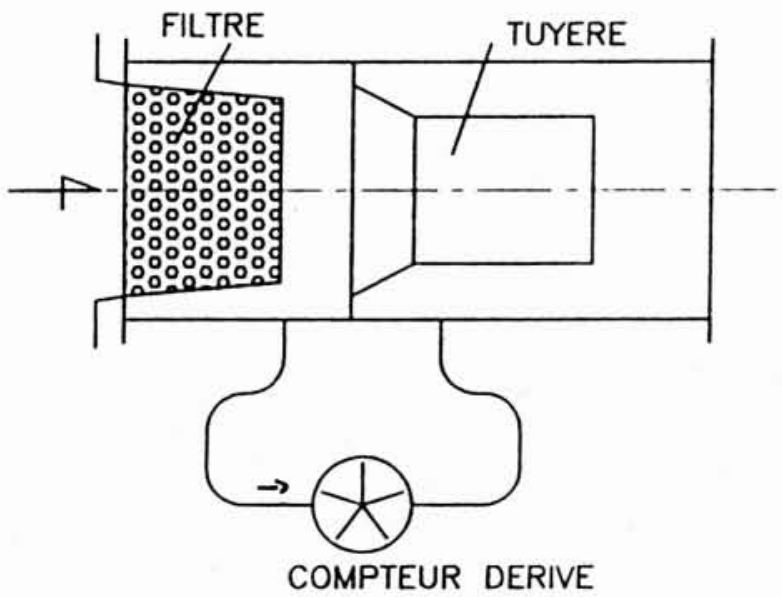

VERTICAL.

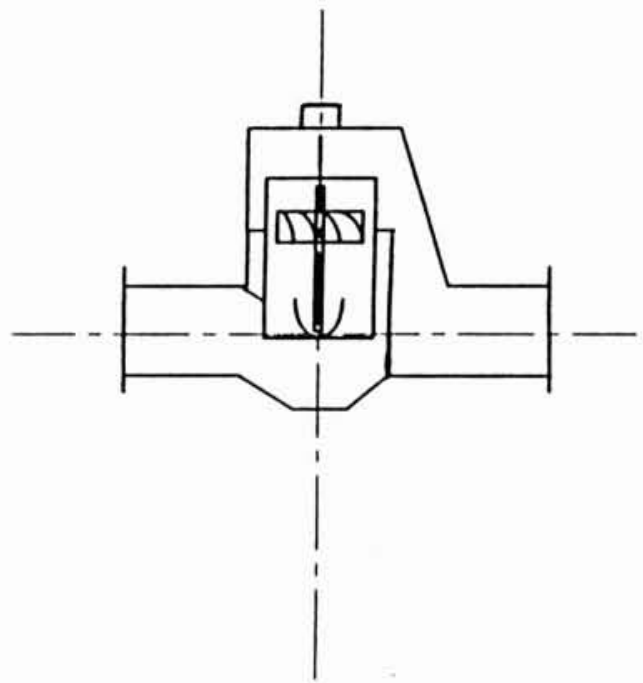

COMPTEUR COMBINÉ

VALVE

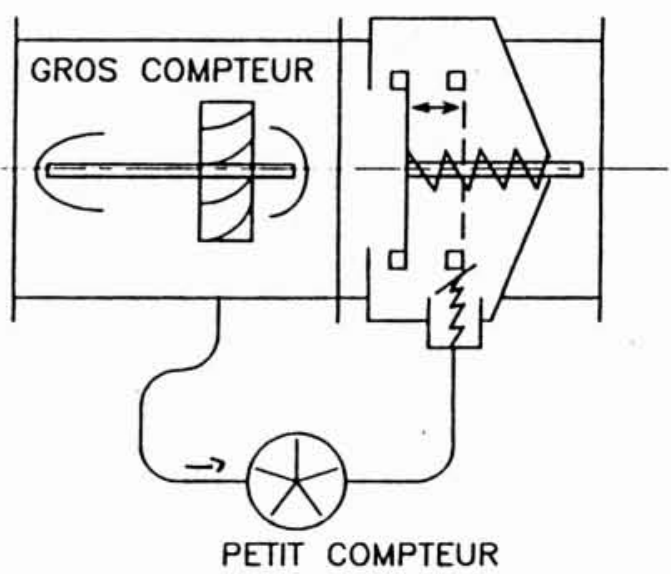

3 bis. Compteurs mécaniques, gros diamètres. 


\subsection{Compteurs WOLTMAN}

Le principe de mesure de vitesse par hélice d'axe parallèle à l'écoulement est mis en œuvre dans le moulinet, ledébitmètre turbine pour hydrocarbures ou le compteur Woltman pour eau. Ce dernier, construit jusqu'au diamètre $500 \mathrm{~mm}$, détermine la vitesse d'eau sur l'ensemble de la section, l'écoulement amont devant être stabilisé par des longueurs droites ou dispositifs spéciaux.

\section{PRINCIPE}

LOI DE FARADAY FORCE ELECTROMOTRICE $U=K \cdot B . \nabla \cdot D$

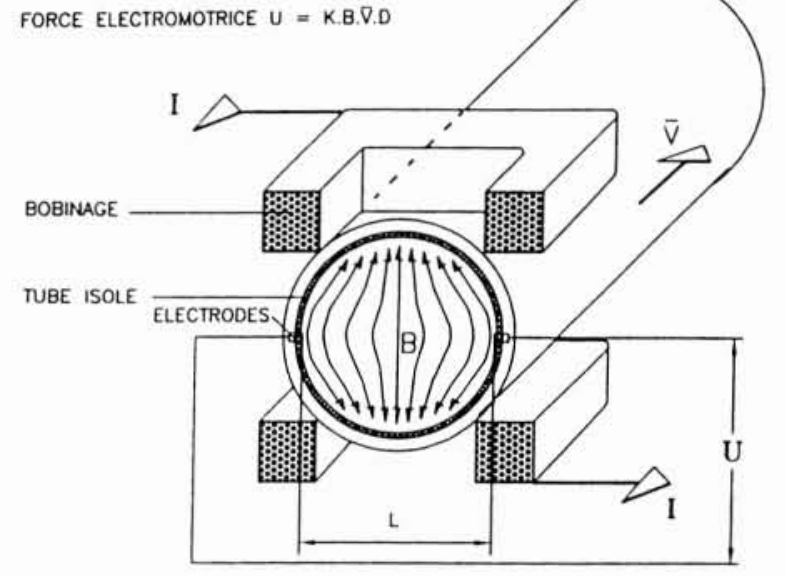

B: ETANT L'INDUCTION MAGNETIQUE

L: LA DISTANCE ENTRE LES ELECTRODES = DIAMETRE INTERIEUR

V: LA VITESSE MOYENNE DU FLUIDE

K: CONSTANTE DU CAPTEUR

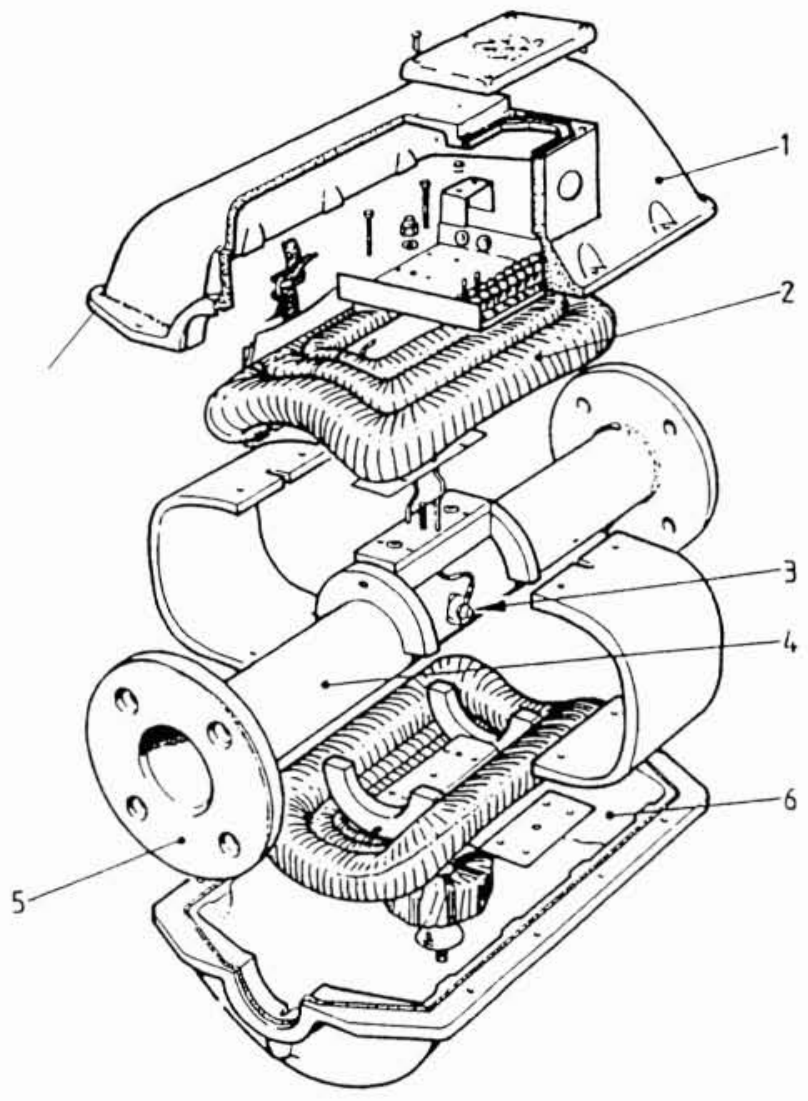

5. Technologie.

1. Enveloppe supérieure.

2. Bobine.

3. Electrodes.

4. Tube de mesure.

5. Revêtement inférieur.

\section{MONTAGE}

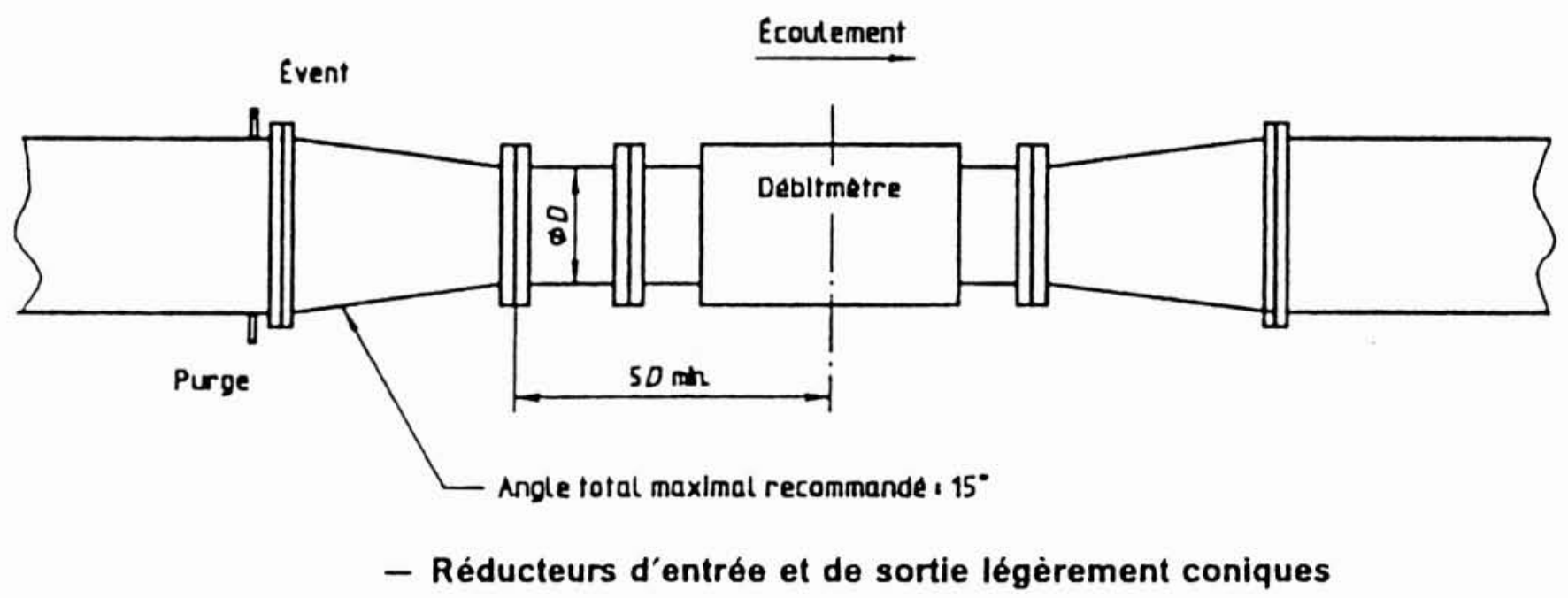

4. Le débitmètre électromagnétique. 
En cas de gros débits ou eaux chargées, son usure est à surveiller. Aussi, bien que sa maintenance soit facilitée lorsque le mécanisme est amovible, on pourra lui préférer un débitmètre sans pièces mobiles.

\section{PRINCIPE}

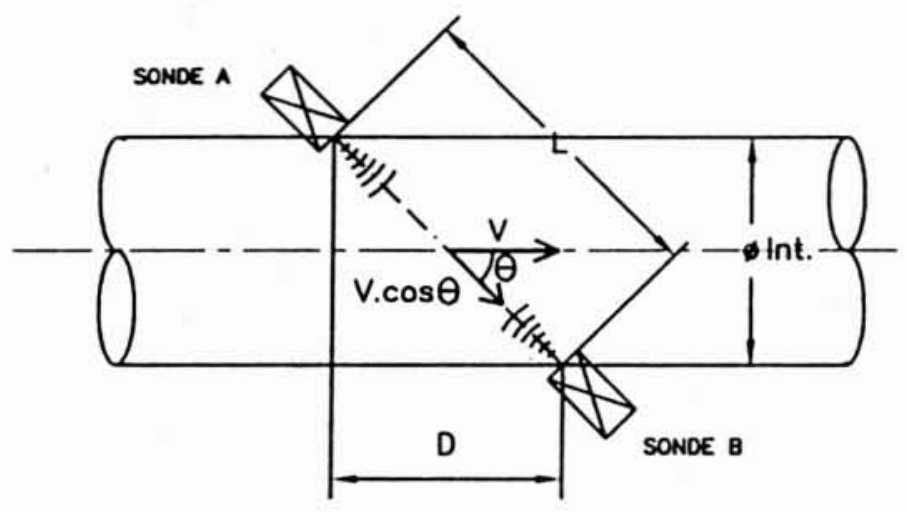

$$
\begin{aligned}
& V \text { US }=\frac{L^{2}}{2 D}\left(\frac{1}{T_{\infty}}-\frac{1}{T_{\text {Q }}}\right) \\
& Q=\frac{\pi \varnothing^{2}}{4} \bar{V}
\end{aligned}
$$

U GEOMETRIE ( L D. Ø ) DOIT ETRE CONNUE.
Le compteur à hélice suspendue comporte un coude à l'entrée pour aider au démarrage à bas débit. Les progrès métrologiques du Woltman axial peuvent conduire à le délaisser, la forme du corps limitant par ailleurs sa taille, comme le compteur à turbine, à $150 \mathrm{~mm}$ de diamètre.

\section{REGLAGE}

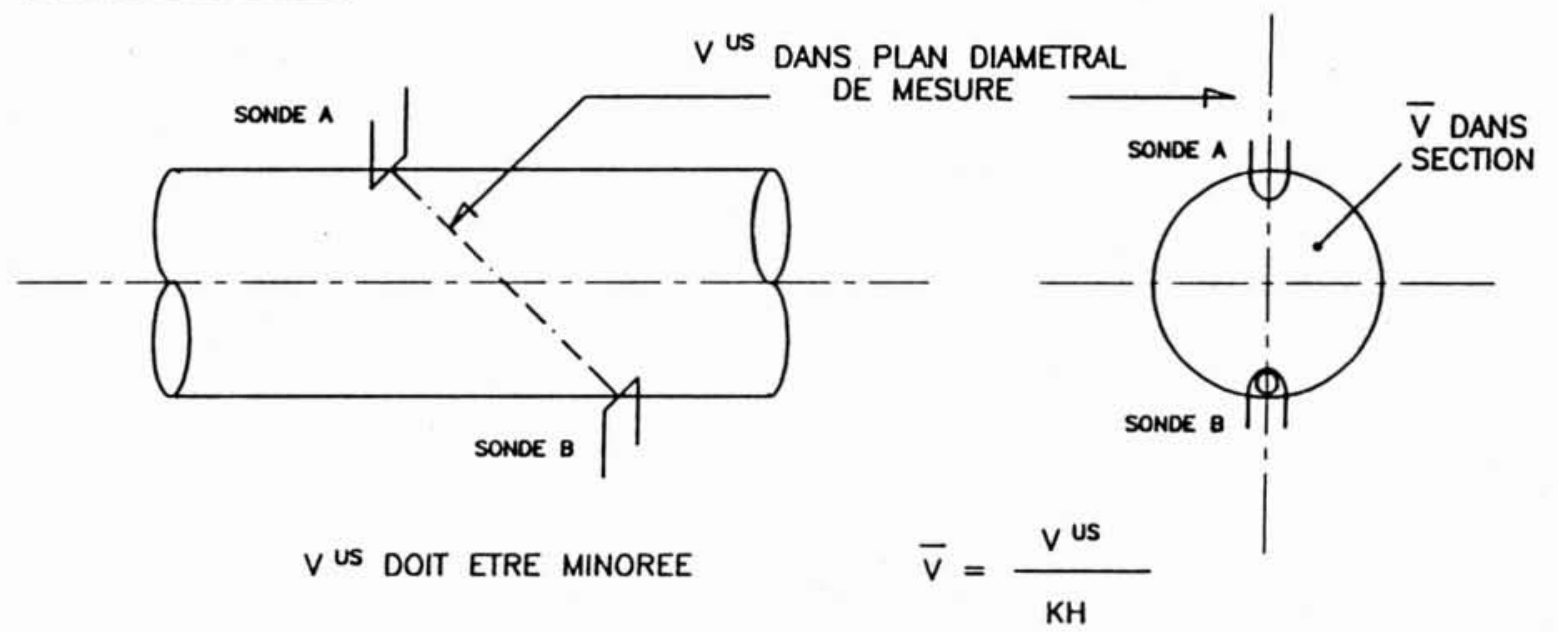

$\mathrm{KH}=$ COEFFICIENT HYDRAULIQUE COMPRIS ENTRE 1.045 et 1.06

\section{TYPES DE SONDES}

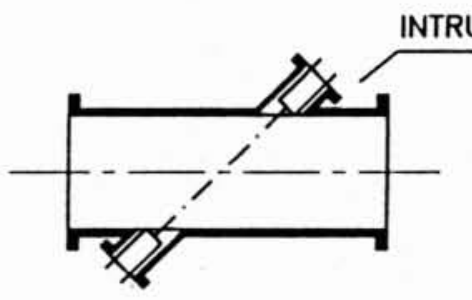

INCUNEES NON DENONTABLES

SUR MANCHETTE

\section{TRUSIVES (MOULIES)}

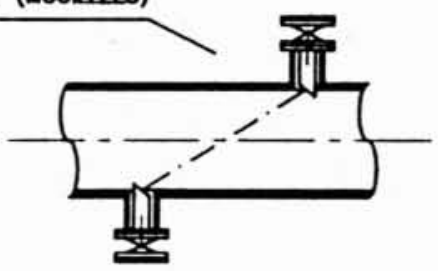

ULTRNTWX

POSE-DEPOSE EN CHWRGE

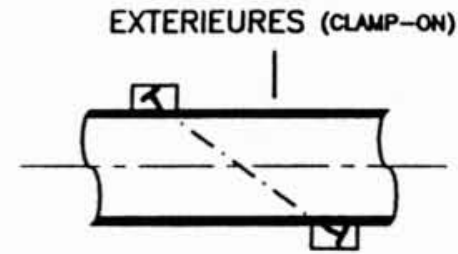

DEBMMETRE PORTNBLE

SUR CONDUITE

6. Le débitmètre à ultrasons à différence de temps de transit monocorde à parcours oblique. 


\section{PRÉSENTATION DES APPAREILS DE MESURE}

\subsection{Compteur combiné}

Assemblage de deux compteurs de tailles différentes, destiné à utiliser leurs étendues de mesure complémentaires au moyen d'une valve d'inversion hydraulique autonome, ce compteur a pu séduire les utilisateurs peu soucieux de calibrage (choix du diamètre en fonction des débits attendus).

Semble moins recherché au moment où son fonctionnement devenait satisfaisant, en raison des contraintes d'exploitation ( 3 appareils en $u n=3$ fois plus de maintenance).

\subsection{Compteur proportionnel}

Sorte d'organe déprimogène dont les orifices de pression différentielle amont aval sont raccordés à un petit compteur dérivé totalisant une faible partie de l'écoulement traversant la section principale.

Permet de mesurer de façon économique des débits élevés, mais précision et fiabilité à surveiller. Non approuvé CEE, il est utilisé en irrigation.

\subsection{Appareil déprimogène}

Dispositif de type venturi ou diaphragme; placé sur une conduite, il permet de déterminer la vitesse moyenne par mesure de pression différentielle.

Il a équipé jadis des conduites de grands diamètres mais n'est plus utilisé sur les réseaux d'eau en raison de sa dynamique réduite résultant de la loi quadratique.

Lorsqu'il est maintenu en service, le suivi de la précision doit inclure le contrôle de l'élément primaire.

Sur une installation neuve, on pourra lui préférer une sonde de Pitot moyennée (annubar) sauf si l'emploi d'orifices normalisés s'impose pour la crédibilité métrologique.

\subsection{Débitmètre électromagnétique (fig. 4 et 5)}

C'est le seul appareil permettant de mesurer la vitesse moyenne sur la totalité d'une section de conduite avec une étendue de mesure importante quels que soient le sens d'écoulement et la qualité de l'eau.

Il a pu effrayer jadis certains utilisateurs en raison de son coût, sa consommation électrique et sa dérive électronique ; ces critiques sont désormais sans fondement.

La grande précision donnée par les constructeurs (quelques millièmes de la valeur mesurée) ne s'impose pas toujours pour le comptage de l'eau vis-à-vis de la directive CEE $( \pm 2 \%)$ et sera difficile à vérifier en exploitation.

\subsection{Débitmètres à ultrasons (fig. 6)}

Techniques très séduisantes par la simplicité et la fiabilité de fonctionnement. La précision de mesure dépend du soin apporté à la pose des capteurs sur le tronçon de conduite dont la géométrie doit être entièrement relevée.

Il ne paraît actuellement utilisé que sur des équipements dont l'installation est bon marché (monocorde adapté sur conduite), provisoire (sondes extérieures) ou sur les grands diamètres. Une baisse de prix de l'électronique en favoriserait la diffusion.

\subsection{Débitmètres à insertion}

Méthode de mesure permettant de déterminer la vitesse moyenne dans une section de conduite par relevé de certaines vitesses locales au moyen de cannes de divers types (Pitot, moulinet, électromagnétique).

Toujours économique, il peut s'avérer précis et crédible selon l'expérience des opérateurs.

\section{Conclusion}

Si l'utilité du comptage ne se discute plus, la qualité des mesures doit être un souci permanent.

Bien qu'il existe de nombreux matériels fiables, précis, adaptés aux besoins, la mesure des débits ou volumes d'eau peut occasionner, de nos jours encore, certaines difficultés dont les conséquences économiques dépassent de beaucoup le coût des appareils.

Il faut donc apporter un soin particulier au choix des débitmètres ou compteurs d'eau en opérant selon des critères économiques, techniques et métrologiques. 